\title{
Latest Results from VERITAS: Gamma 2016
}

\author{
Jamie Holder $^{1, a)}$ and the VERITAS Collaboration ${ }^{\text {b) }}$ \\ ${ }^{1}$ Department of Physics and Astronomy and the Bartol Research Institute, University of Delaware, DE 19716, USA. \\ a)jholder@physics.udel.edu \\ b)URL: http://veritas.sao.arizona.edu
}

\begin{abstract}
The VERITAS imaging atmospheric Cherenkov telescope array has been observing the northern TeV sky with four telescopes since summer 2007. Over 50 gamma-ray sources have been studied, including active and starburst galaxies, pulsars and their nebulae, supernova remnants and Galactic binary systems. We review here some of the most recent VERITAS results, and discuss the status and prospects for collaborative work with other gamma-ray instruments, and with multimessenger observatories.
\end{abstract}

\section{INTRODUCTION}

VERITAS (Figure 1) is an imaging atmospheric Cherenkov telescope array, now entering its tenth year of operations. The array consists of four identical telescopes, located at the Fred Lawrence Whipple Observatory (FLWO) in Arizona, each with a $12 \mathrm{~m}$ diameter tessellated reflector. Cherenkov light from gamma-ray and cosmic-ray initiated particle cascades is focused by the telescope reflectors onto 499-pixel photomultiplier tube (PMT) cameras, which cover a $3.5^{\circ}$ field of view.

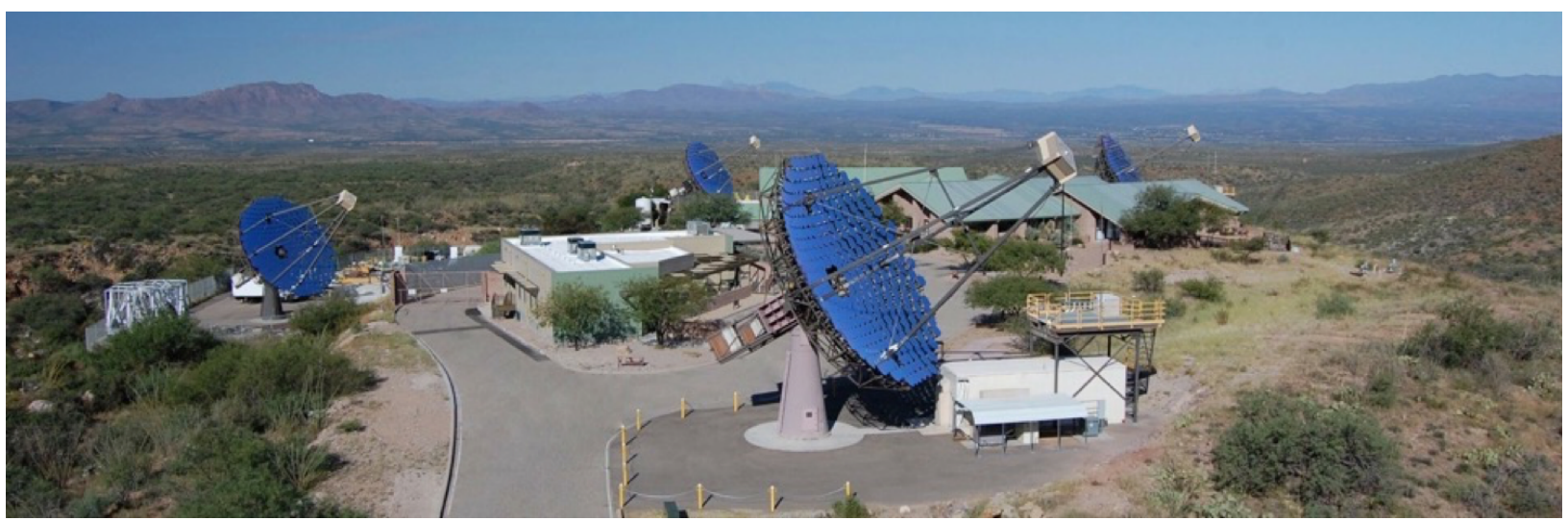

FIGURE 1. The VERITAS array in its current (2016) configuration.

The array has undergone two major upgrades over the past decade. The first, in 2009, involved the relocation of the original prototype telescope to a more favorable location, resulting in an approximate diamond-shaped layout with sides of $\sim 100 \mathrm{~m}$. This improved the angular reconstruction capabilities, and enhanced the sensitivity of the array. In 2012, a major overhaul of the telescope electronics saw the installation of new trigger systems, and the replacement of all of the photosensors with higher quantum efficiency (super bialkali photocathode) PMTs. This again improved sensitivity, and led to a dramatic enhancement of the low energy response. Thanks to these upgrades, and to improvements in analysis and calibration tools, VERITAS now detects a source with $1 \%$ of the steady Crab Nebula flux in under 25 hours; less than half of the exposure required in the original array configuration. Of similar importance is the increase in duty cycle provided by moonlight observations. VERITAS now commonly conducts observations 
with the lunar disk up to $50 \%$ illuminated, including with reduced PMT voltage for higher illuminations. Moonlight observations account for as much as $\sim 40 \%$ of the total annual observing yield [1], which averages approximately 1300 hours per year.

Figure 2 shows the differential sensitivity of VERITAS for three different epochs. Further details are available in [2].

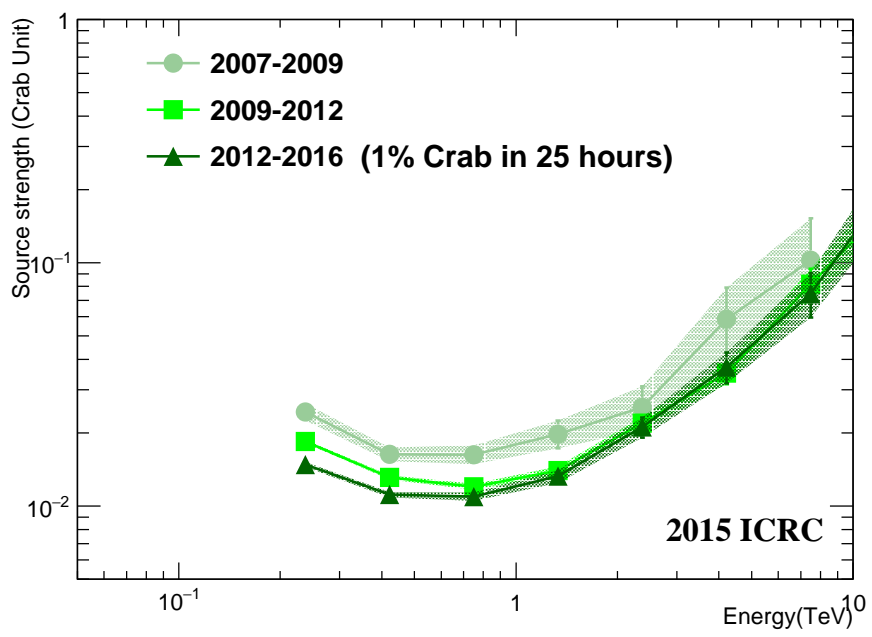

FIGURE 2. VERITAS sensistivity for the three different configurations of the instrument.

The VERITAS source catalog (Figure 3) now stands at 56 sources, and is composed of eight different source classes. To make the most efficient use of limited observing time, the balance of the observing plan has shifted from attempts to detect new sources, to precision measurements requiring deep exposures, or observations of variable sources during exceptional high flux states. We summarize here some of the most interesting results from the past two years of VERITAS operations.

\section{GALACTIC SOURCES}

\section{Supernova Remnants}

Supernova remnants (SNRs) are key targets for precision measurements with VERITAS, since the details of their gamma-ray morphology and spectra can be used to inform and constrain models of cosmic-ray acceleration and diffusion near the source. Exposures on Tycho's SNR, IC 443 and Cassiopeia A have all more than doubled since the original VERITAS publications, and much of the more recent data has been taken with the most sensitive configuration of the observatory.

Tycho's SNR is the remnant of a historical Type Ia supernova, well-studied over all wavelengths. The relative simplicity of the environment around this remnant makes it a favored system for theoretical models (e.g. [3, 4, 5, 6]). Gamma-ray emission from the source was discovered by VERITAS in 2011, at the level of $0.9 \%$ of the Crab Nebula flux [7]. The broadband spectral energy distribution (SED) has been interpreted within both hadronic and leptonic scenarios. The updated VERITAS spectrum [8], shown in Figure 4, extends the published measurement down in energy from $\sim 800 \mathrm{GeV}$ to $\sim 400 \mathrm{GeV}$, and, while consistent, can be fit with a softer power-law than the previous result $\left(\Gamma=2.92 \pm 0.42_{\text {stat }}\right)$. This may imply a steeper spectrum of accelerated particles than predicted by standard diffusive shock acceleration models [9].

Like Tycho, Cas A is a young remnant, unresolved at TeV energies, but the relatively high gamma-ray flux allows for much more precise spectral measurements. Figure 4 shows the most recent VERITAS spectrum [10], calculated using over 60 hours of data collected over 6 years (three times the originally published exposure). When combined 


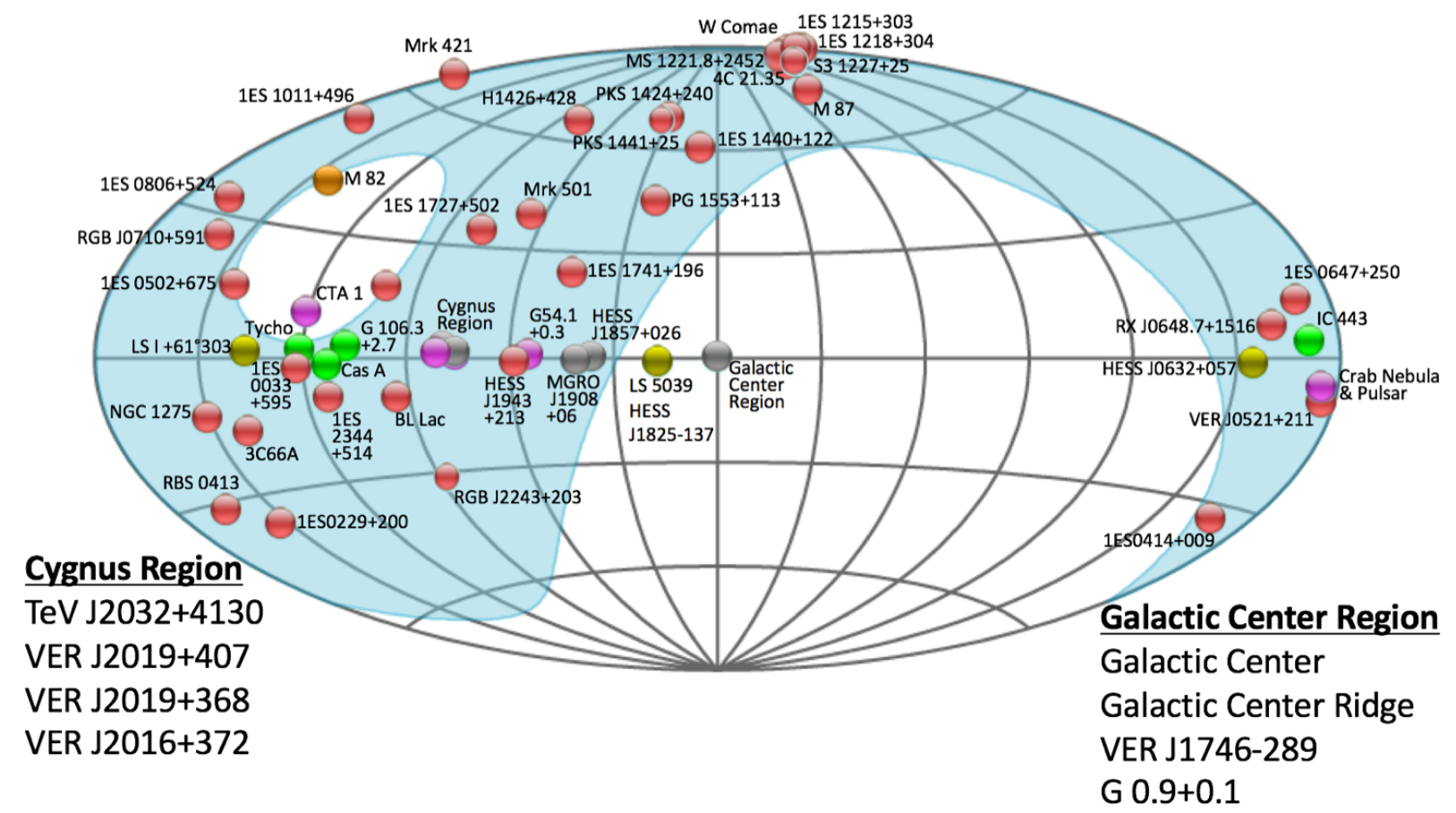

FIGURE 3. The VERITAS source catalog, in Galactic coordinates, as of July 2016. Shaded regions indicate visibility to VERITAS above $55^{\circ}$ elevation. Figure modified from TeVCat (http://tevcat.uchicago.edu).

with Fermi-LAT results at lower energies, a broken power-law fit is favored. The centroid of the emission is consistent with an unresolved source at the the center of the remnant, and with other measurements at $\mathrm{TeV}$ and $\mathrm{GeV}$ energies.

IC 443 is an older remnant (3-30 kyr) expanding into an extremely inhomogeneous environment. It has been established as a hadronic accelerator, through the measurement of a pion bump signature in the $\mathrm{MeV}-\mathrm{GeV}$ range [11]. The initial TeV gamma-ray detections $[12,13]$ identified a single site of emission co-located with dense molecular clouds, with which the SNR is interacting. Updated results from VERITAS now resolve emission from the entire shell of the remnant, as illustrated in Figure 5 [14]. Also shown is the $>1 \mathrm{GeV}$ map from Fermi-LAT, which correlates well with the $\mathrm{TeV}$ emission [15]. The spectrum steepens from the GeV to the TeV regime (from $\sim 2.3$ to $\sim 2.9$ ), and this behaviour is remarkably uniform across the entire remnant, despite the widely varying environmental conditions and integrated flux values. The interpretation of these results is ongoing, but IC 443 clearly provides a valuable laboratory for the exploration of particle acceleration and diffusion over a wide range of environmental conditions in a single object.

\section{Gamma-ray Binary Systems}

Precision measurements of gamma-ray binary systems with VERITAS have also been conducted over the almost decade-long baseline. These attempt to provide dense and complete sampling of the gamma-ray lightcurve over all orbital phases, as well as to monitor the systems for unusual flux states. New results on HESS J0632+057 are presented at this conference [16]. The full 315-day orbit is now sampled by VERITAS observations, providing clear detections and spectral measurements over all phases in which emission is detected (Figure 6). Also shown are observations of LS I+61 303 which, in 2014, revealed exceptionally bright TeV flares, reaching 30\% of the Crab Nebula with variability on daily timescales [17]. Plans for the coming seasons include monitoring of PSR J2032+4127 as it approaches periastron in 2017-2018. This system is a recently identified 20-30 year-period pulsar-Be star binary co-located, and possibly associated, with $\mathrm{TeV} 2032+4130$ [18]. 

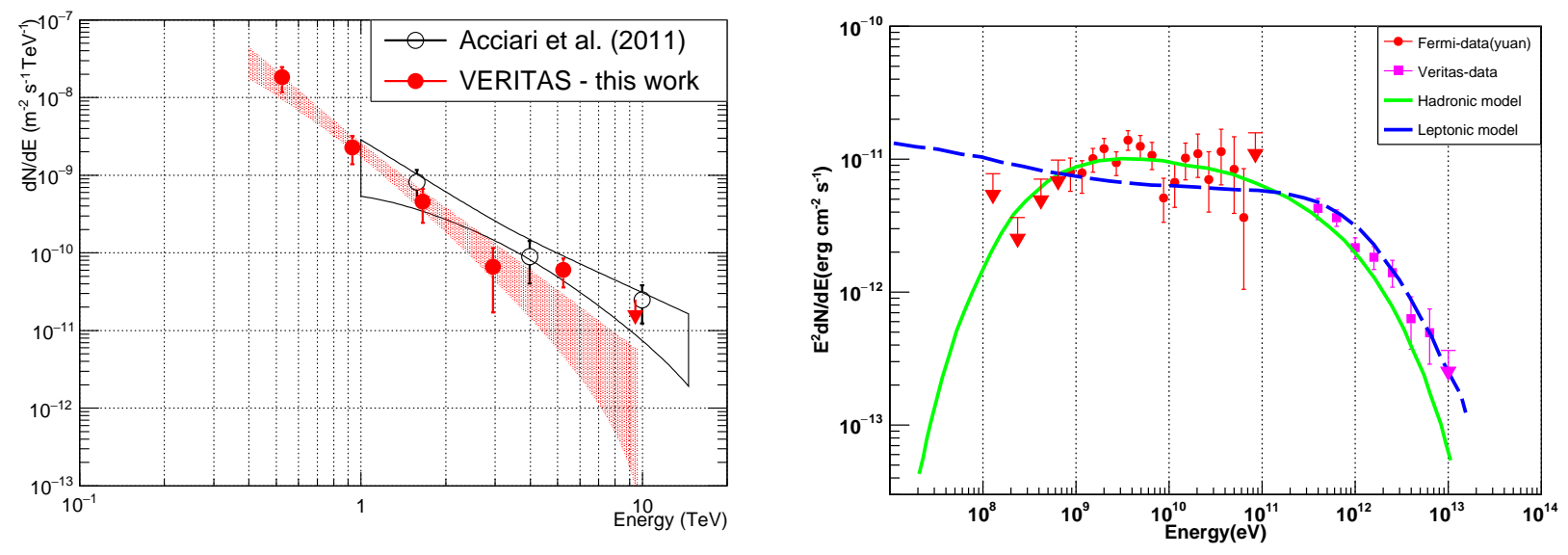

FIGURE 4. VERITAS spectra of the Tycho (left) and Cas A (right) supernova remnants.

\section{The Galactic Center Region}

VERITAS results on a deep exposure ( 85 hours) of the Galactic Center region were also recently published [19]. With an average elevation angle of $\sim 30^{\circ}$, the energy threshold for this analysis is $2 \mathrm{TeV}$, complementing studies by H.E.S.S. Point-like emission from the direction of both SgrA* and the composite SNR G0.9+0.1 has been measured. When these sources are subtracted from the excess maps, residual emission is observed along the Galactic Ridge, including a relatively bright component close to SgrA*, which we label VER J1746-289 (Figure 7).

\section{EXTRAGALACTIC SOURCES}

With its location in the northern hemisphere, much of VERITAS science naturally focuses on the extragalactic sky, and active galactic nuclei (AGN) are the most numerous source class in the catalog. VERITAS has detected 34 AGN, the majority of which are high- or intermediate-frequency peaked BL Lacertae objects (HBLs/IBLs). The remainder are the flat spectrum radio quasars (FSRQs), PKS $1222+216$ and PKS $1441+25$, the nearby radio galaxies M87 and NGC 1275 , and the starburst galaxy M82. Multiwavelength monitoring programs provide contemporaneous measurements of the broadband SEDs of many of these sources, in particular through close collaboration with the Swift X-Ray Telescope team. VERITAS blazar results are covered in more detail elsewhere in these proceedings [20, 21], as is their use as probes of the extragalactic background light (EBL) and intergalactic magnetic fields [22]. Here we highlight just a couple of the most exciting recent extragalactic source results.

\section{PKS 1441+25}

PKS $1441+25$ is one of only two FSRQs detected by VERITAS [23] and, at a redshift of $z=0.939$, is the second-most distant source in the very high energy sky. Observations in April 2015, triggered by alerts from Fermi-LAT [24] and MAGIC [25], allowed a precise measurement of the emission during a high state, in which the flux reached $\sim 5 \%$ of the Crab Nebula flux above $85 \mathrm{GeV}$. The spectral index, $\Gamma=-5.4 \pm 0.5$, is the softest yet measured by VERITAS for any source, corresponding to an intrinsic index of $\Gamma=-3.4 \pm 0.5$ after correction for absorption by the EBL (Figure 8). No emission is detected above $\sim 200 \mathrm{GeV}$, illustrating the importance of the energy threshold reduction provided by the 2012 camera and trigger upgrades for extragalactic science. Gamma-ray opacity arguments, as well as multiwavelength correlations, indicate that the origin of the emission must lie far from the center of the AGN, outside of the broad line region. The extreme distance of the source, coupled with the highly significant measurement of its spectrum, allow to place constraints on the intensity of the near-ultraviolet to near-infrared EBL which are comparable to those derived from stacking multiple sources with lower redshifts, and agree with EBL estimates from galaxy counts. 

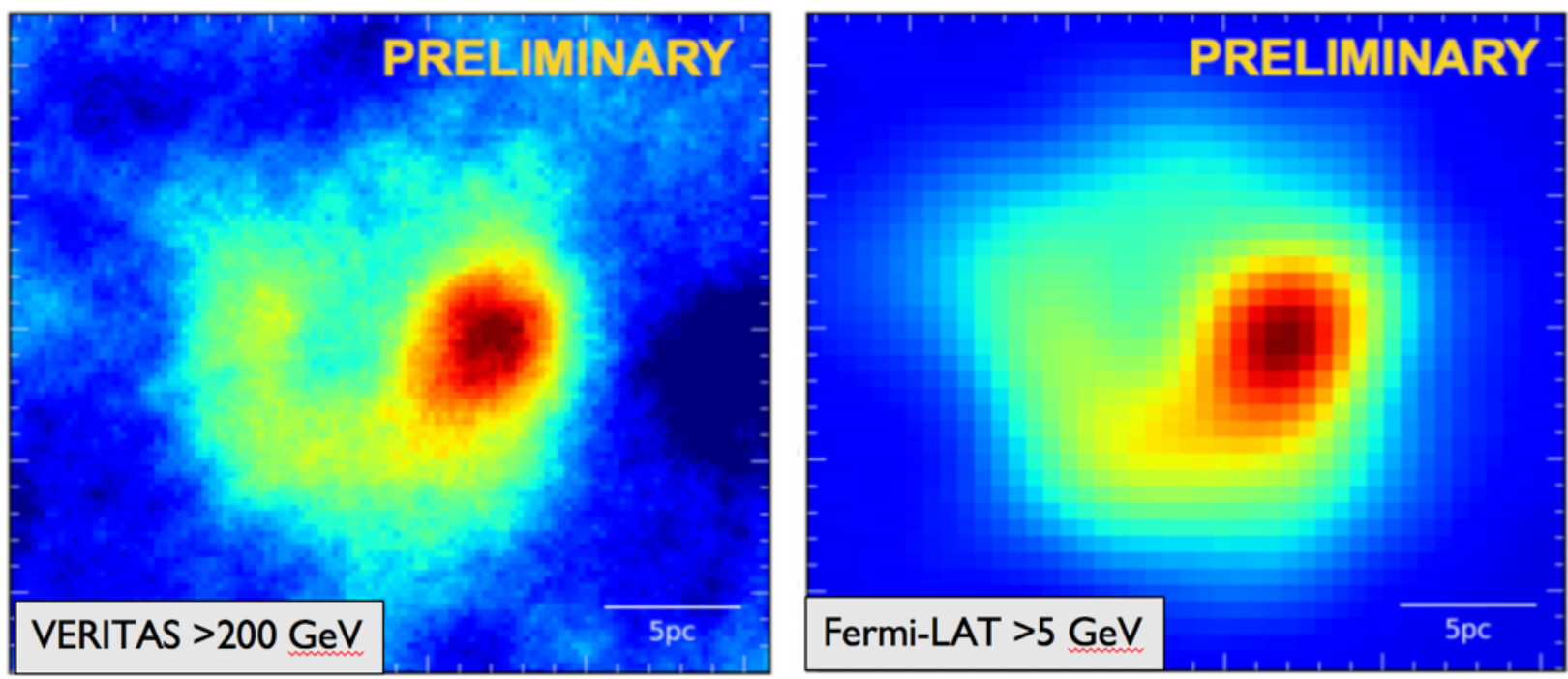

FIGURE 5. VERITAS and Fermi-LAT skymaps of IC 443, showing resolved emission over the full extent of the supernova remnant.

\section{ES 1959+650}

1ES 1959+650 was among the first TeV blazars to be detected [26] and holds particular interest as the clearest example of a TeV source which has exhibited "orphan" flaring - a prominent gamma-ray flare with no X-ray counterpart [27]. The source has been in an extended active state since summer 2015, and VERITAS observed flares at the level of the Crab Nebula flux in fall 2015 [28]. Further flaring activity in 2016 triggered additional VERITAS monitoring, revealing the source to be in an unprecedented high state, with flares exceeding 2.5 Crab (Figure 8). Cooperation and communication between the northern hemisphere gamma-ray instruments, Fermi-LAT and Swift places this among the best-sampled extreme blazar flaring events, with observations often conducted multiple times within a 24-hour period.

\section{OTHER TOPICS}

Gamma-ray astronomy is an important and growing field, but imaging atmospheric Cherenkov telescopes (IACTs) provide the means to study more than gamma-ray emission from specific astrophysical objects. We summarize some recent updates from VERITAS below.

\section{Dark Matter Searches}

The most recent update on the search for gamma rays from WIMP annihilation in dwarf spheroidal galaxies with VERITAS was presented at the 2015 ICRC [29]. As the exposure for targets with the highest estimated luminosity due to dark matter annihilation approaches $~ 100$ hours, further deep observations of individual targets provide diminishing returns, and are prone to systematic biases. An alternative approach, which offers considerable improvements, is to combine the data from multiple sources, weighting individual events according to their energies, locations, and the properties of the instrumental and astrophysical backgrounds [30]. Using this method, the combination of over 200 hours of observations, spread over 4 dwarf spheroidal galaxies, has been used to provide improved limits to the annihilation cross-section.

\section{Cosmic-Ray Electrons}

The cosmic-ray electron/positron spectrum provides a direct measurement of cosmic-ray acceleration and diffusion in our local Galactic neighbourhood. Interest in this area has grown with the observation, by multiple instruments, of a positron component which increases up to an energy of $\sim 200 \mathrm{GeV}$. While they are unable to easily discriminate 

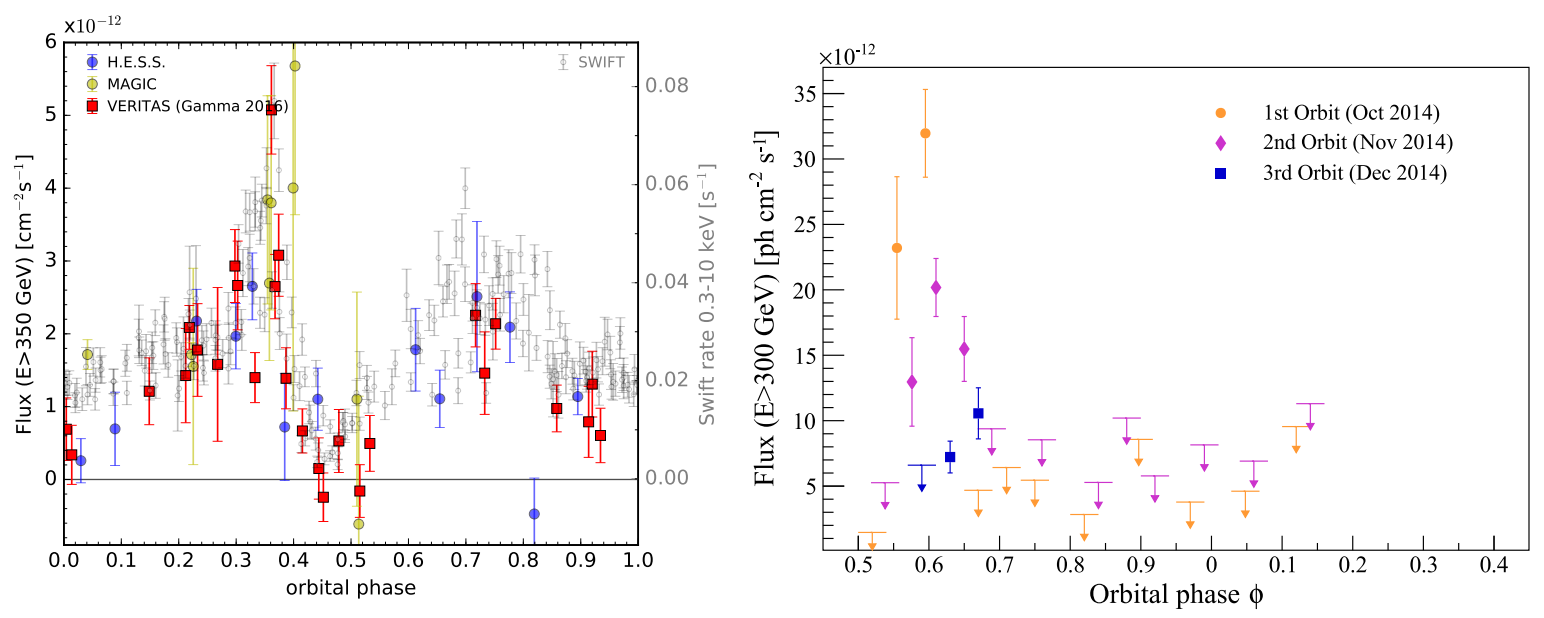

FIGURE 6. VERITAS observations of HESS J0632+057 (left) and bright flaring activity from LS I+61 303 in 2014 , reaching $30 \%$ of the Crab Nebula flux (right).

electrons and positrons, the large effective collection area of IACTs allows to extend measurements of the spectrum of cosmic-ray electrons beyond those energies probed by AMS and Fermi-LAT. VERITAS has now measured the combined electron-positron spectrum up to a few $\mathrm{TeV}$ [31]. The results are consistent with those from H.E.S.S., and the spectrum is best fit with two power-laws with a break energy of $710 \pm 40 \mathrm{GeV}$ (Figure 9).

\section{Optical Transients}

With their large mirror area, IACTs are powerful optical instruments, and probe a unique temporal parameter space. VERITAS has recently developed an efficient method to search for ultra-fast optical transients from astronomical objects, sensitive to nanosecond pulses with fluxes as low as $\sim 1$ photon $\mathrm{m}^{-2}$. We have applied this technique to search for optical pulses in serendipitous archival observations of KIC 8462852, a star with a peculiar variability pattern which has been suggested as an exceptional target for SETI searches [32]. Unsurprisingly, no emission was detected, but the technique is now established, and can be applied to searches of the extensive VERITAS data archives for brief optical transient events.

\section{NEW PARTNERS}

VERITAS has developed ongoing partnerships with many collaborations around the world, working both in gammarays and at other wavelengths, as well as with multi-messenger astroparticle physics observatories. Collaborative efforts with two relatively new facilities have recently enhanced the scientific program of VERITAS.

\section{VERITAS and IceCube}

The IceCube observatory has detected an isotropic flux of TeV-PeV astrophysical neutrinos, whose origin remains unclear [33]. IACT arrays, including VERITAS, can attempt to identify a gamma-ray counterpart, or to constrain the properties of the neutrino source population. VERITAS has made follow-up observations of 18 well-located IceCube events; those containing muon tracks, with $<1^{\circ}$ angular uncertainty (Figure 10). No significant emission has been detected, and upper limits are typically a few $\%$ of the Crab Nebula flux [34].

The sources of astrophysical neutrinos may be variable, or have a variable component. In this case, prompt follow-up observations of any neutrino event could be the key to the discovery of a gamma-ray counterpart. VERITAS and MAGIC have both taken part in a long-running program, responding to potential neutrino flares originating from the direction of a list of candidate sources (mostly blazars) [35]. With the identification of a high energy astrophysical neutrino flux, more stringent conditions can now be applied to ensure that follow-up observations are targeting the locations of astrophysical neutrinos (as opposed to fluctuations in the background of atmospheric neutrinos). Using 


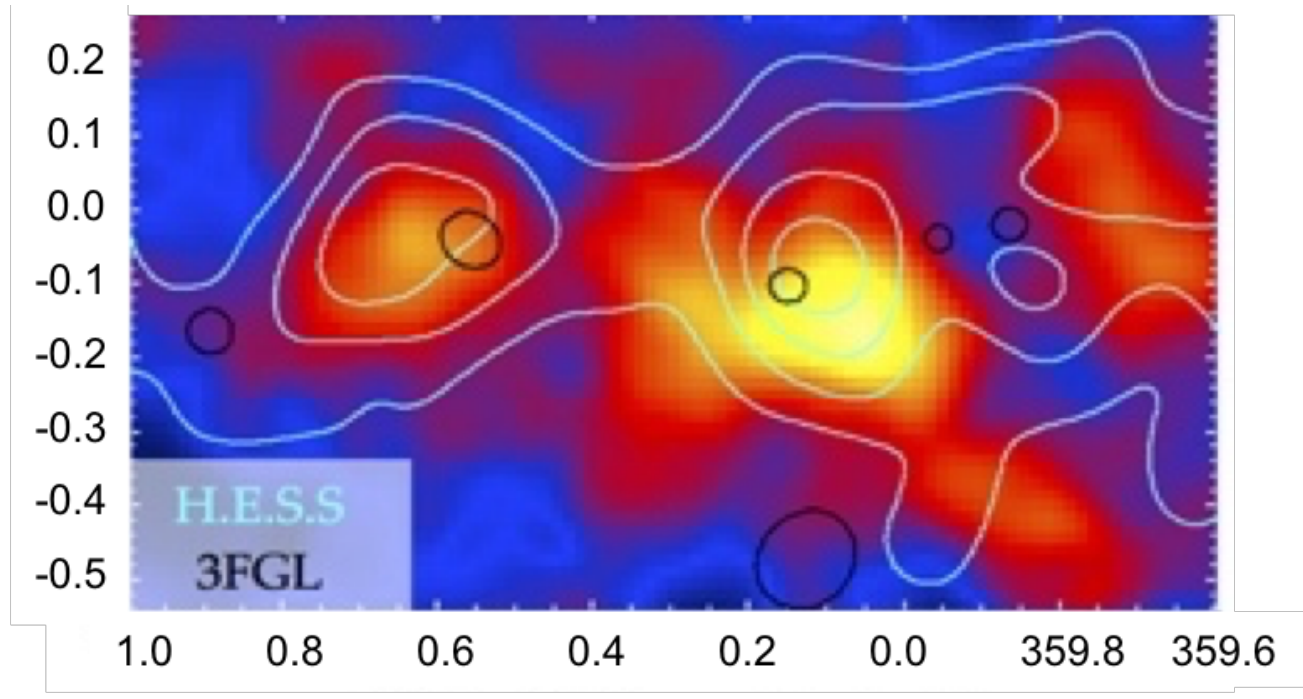

FIGURE 7. VERITAS map of the Galactic Center region (in Galactic coordinates), with unresolved emission from the direction of SgrA* and SNR G0.9+0.1 subtracted. Emission contours from H.E.S.S. are also shown (cyan), along with error ellipses for Fermi-LAT sources (black). See [19] for details.

infrastructure developed for gamma-ray burst studies, IceCube is now providing prompt Gamma-ray Coordinates Network (GCN) alerts for high-energy starting events (HESE) through the astrophysical multimessenger observatory network (AMON). VERITAS received its first alert in April 2016, with observations starting after 193 seconds. Almost all of the data were taken under bright moonlight conditions. Unfortunately, a revised location, issued 18 hours after the initial alert, placed the neutrino point of origin outside of the field of view of the VERITAS observations. The revised location was targeted by VERITAS the following night, but showed no excess. VERITAS flux upper limits were posted as a GCN circular. The prompt response provided a useful demonstration of the capabilities of the alert program, and observations will continue over the coming observing seasons.

\section{VERITAS and HAWC}

The most recent partner facility to be fully commissioned is the High Altitude Water Cerenkov (HAWC) observatory, which observes the northern hemisphere very high energy gamma-ray sky continuously from its location in Mexico. HAWC is able to provide alerts to the TeV observatories, which allows prompt follow-up of extreme flaring events (e.g. [36]). The much higher instantaneous sensitivity of IACTs can then be used to study such events in detail and VERITAS, located at almost the same longitude as HAWC, is particularly well-suited to this task.

The wide-field survey capabilities of HAWC provide another promising avenue to new science. HAWC has recently released a preliminary skymap based on 341 day's live-time with almost the full detector in operation. Further results were shown in detail at this meeting [37,38]. While the VERITAS field-of-view $\left(\Phi=3.5^{\circ}\right)$ is much smaller than HAWC, VERITAS has been in operation since 2007, and has archival observations of a significant fraction of the celestial sphere (Figure 11). This includes exposures, totalling 100 hours, which cover at least 10 of the sources and source candidates in the preliminary HAWC catalog which do not have an existing $\mathrm{TeV}$ counterpart. A consistent re-analysis of these data using the most recent analysis tools is in progress, and the results will be presented at future meetings. We note that there exists some overlap with the VERITAS survey of the Cygnus region, an update to which was presented here [39].

Dedicated follow-up observations have been made of two particularly interesting regions identified by HAWC. The first is the region close to SNR G54.1+0.3/PSR 1930+1852. VERITAS detected a gamma-ray counterpart to this object, VER J1930+188, in 2010 [40]. The emission is point-like, with a spectral index of $\Gamma=-2.39 \pm 0.23_{\text {stat }} \pm 0.30_{\text {sys }}$, and is likely associated with the pulsar wind nebula. The HAWC view of this region displays emission from the location of the VERITAS source (2HWC J1930+188), along with two further sources located $0.8^{\circ}$ (2HWC J1927+187) and $1.2^{\circ}$ (2HWC J1928+178) from PSR 1930+1852. Both of these excesses are close to relatively high spindown luminosity pulsars (PSR J1928+1746 and PSR B1925+18.8), which may provide plausible candidates for the 

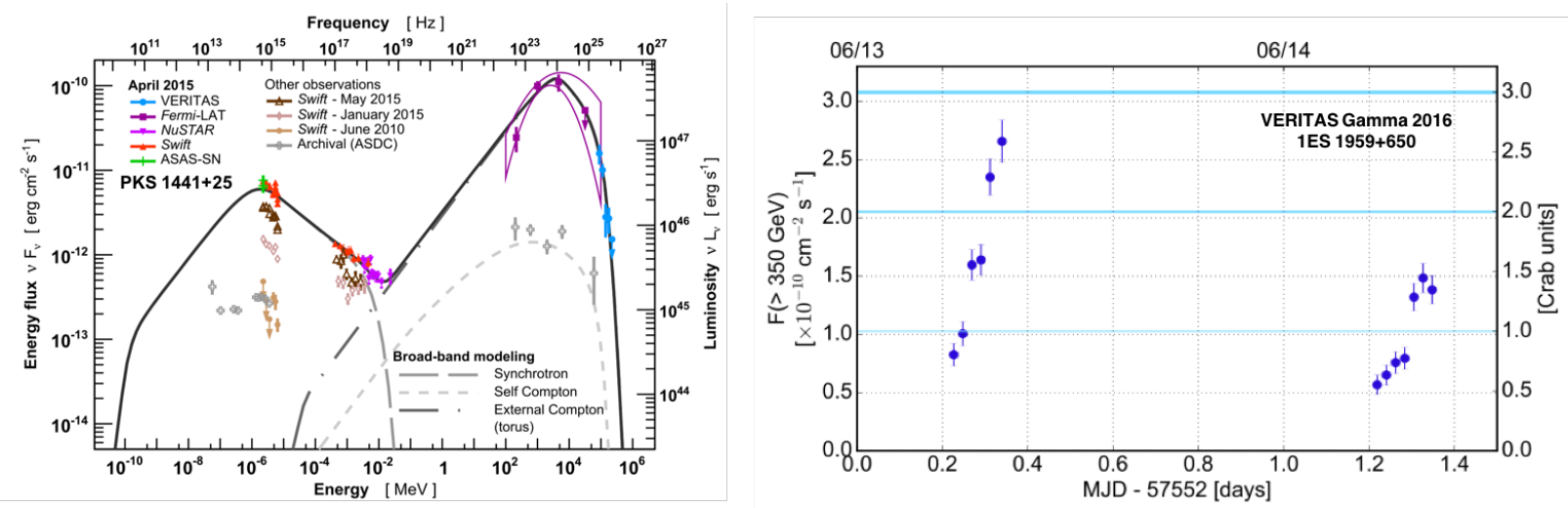

FIGURE 8. The broad-band SED of the distant FSRQ, PKS 1441+25 (left), and (right) a portion of the VERITAS lightcurve for 1ES 1959+650 during an active period in June 2016.

HAWC emission. We note that the original VERITAS publication already placed limits to point-like emission from PSR J1928+1746 at the level of $F(>1 \mathrm{TeV})<2.6 \times 10^{-13} \mathrm{~cm}^{-2} \mathrm{~s}^{-1}$.

VERITAS has 27 hours of archival data on this region, taken in standard "wobble" mode with $0.5^{\circ}$ offset around G54.1+0.3. All of these data were recorded prior to 2009 (before any of the upgrades to VERITAS were implemented), and half are with only 3 of the 4 telescopes in operation. We have supplemented these data with an additional 12 hours of recent observations (June 2016), targeting a point roughly equidistant from the 3 HAWC sources. The resulting skymap, for the complete dataset, is shown on the left in Figure 12. VER J1930+188 is re-detected, as we would expect, but there is no evidence for emission from either of the new HAWC sources. Studies are ongoing, but the non-detection may be explained by a combination of effects: for example, the limited sensitivity of the archival data for sources with large offsets, and/or the influence of source characteristics such as spatial extension and hard spectra.

Dedicated VERITAS observations have also been performed this summer of another of the preliminary HAWC source candidates, 2HWC J1953+294. The VERITAS dataset in this case totals 37 hours, and reveals a statistically significant gamma-ray source located within the HAWC source contours (Figure 12, right). The emission is coincident with the PWN DA $495($ G65.7+1.2) and its central object, WGA J1952.2+2925. Karpova et al. [41] have argued that, despite the lack of observed pulsations, the Fermi-LAT source 3FGL J1951.6+2926 is likely the counterpart of the pulsar in this system, and not of the PWN. The addition of VHE measurements from HAWC and VERITAS to the broadband SED will soon help to determine the nature of the high energy emission from this interesting object.

\section{SUMMARY}

VERITAS continues to operate smoothly, and the instrument is currently in its most sensitive configuration to-date. Ongoing analysis developments promise further incremental improvements in the coming years, while new scientific opportunities are presented by the introduction of HAWC, IceCube, and other facilities. Operational funding for VERITAS is secure, and the collaboration plans to continue observing with the array until at least 2019, with a 10-year anniversary workshop planned for 2017. The construction of the prototype mid-size Schwarzchild-Couder Telescope [42] at the FLWO provides a firm link to CTA, and to the future of ground-based gamma-ray astronomy.

\section{ACKNOWLEDGEMENTS}

This research is supported by grants from the U.S. Department of Energy Office of Science, the U.S. National Science Foundation and the Smithsonian Institution, and by NSERC in Canada. We acknowledge the excellent work of the technical support staff at the Fred Lawrence Whipple Observatory and at the collaborating institutions in the construction and operation of the instrument. The VERITAS Collaboration is grateful to Trevor Weekes for his seminal contributions and leadership in the field of VHE gamma-ray astrophysics, which made this study possible. 


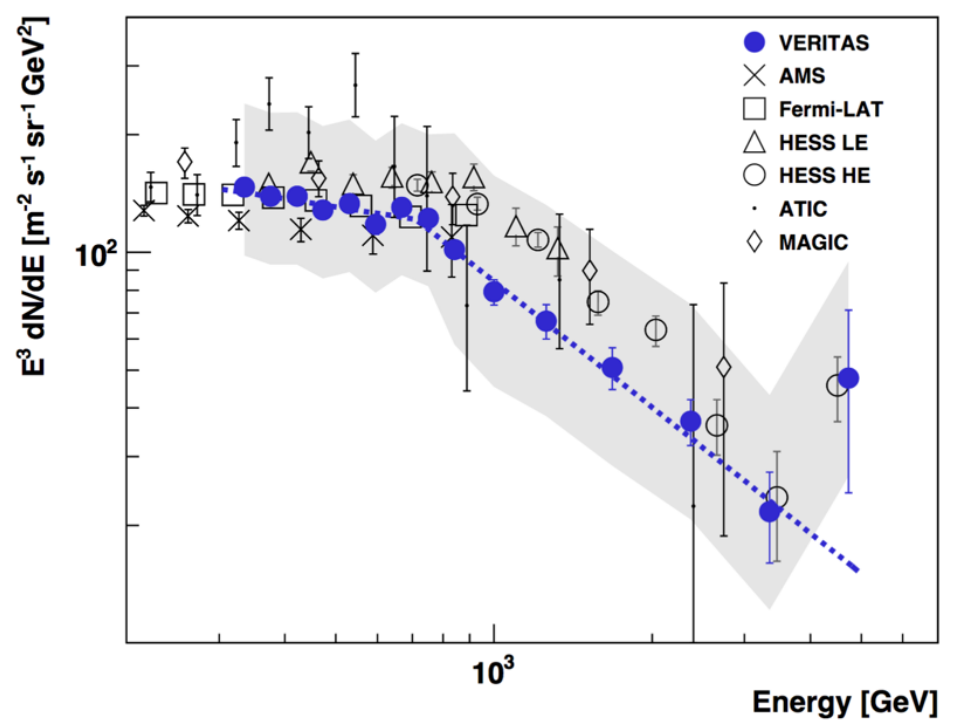

FIGURE 9. VERITAS cosmic-ray electron spectrum (blue circles), fit with two power-laws with a break energy of $710 \pm 40 \mathrm{GeV}$. The gray band shows the systematic uncertainty on the VERITAS measurement [31].

\section{REFERENCES}

[1] Archambault, S., et al., ApJ, 808, 110, 2015

[2] Park, N., for the VERITAS Collaboration, in Proc. 34 $4^{\text {th }}$ ICRC, (The Hague, The Netherlands, 2015), arXiv:1508.07070

[3] Morlino, G., \& Caprioli, D., A\&A, 538, A81, 2012

[4] Atoyan, A., \& Dermer, C. D., ApJL, 749, L26, 2012

[5] Berezhko, E. G., Ksenofontov, L. T., Voelk, H. J., ApJ, 763, 14, 2013

[6] Slane, P., et al., ApJ, 783, 33, 2014.

[7] Acciari, V. A., et al., ApJL, 730, L20, 2011.

[8] Park, N., for the VERITAS Collaboration, in Proc. 34 $4^{\text {th }}$ ICRC, (The Hague, The Netherlands, 2015), arXiv:1508.07068.

[9] Morlino, G., \& Blasi, P., A\&A, 589, A7, 2016.

[10] Kumar, S., for the VERITAS Collaboration, in Proc. 34 $4^{\text {th }}$ ICRC, (The Hague, The Netherlands, 2015), arXiv: 1508.07453

[11] Ackermann, M., et al., Science, 339, 807, 2013.

[12] Albert, J., et al., ApJL, 664, L87, 2007.

[13] Acciari, V. A., et al., ApjL, 698, L133, 2009.

[14] Humensky, B., for the VERITAS Collaboration, in Proc. $34^{\text {th }}$ ICRC, (The Hague, The Netherlands, 2015), arXiv: 1512.01911

[15] Hewitt, J. W., Hays, E. A., Tajima, H., Schmid, J., \& LAT Collaboration, V. C. 2016, American Astronomical Society Meeting Abstracts, 227, 238.10

[16] Schlensted, S. for the VERITAS Collaboration. This conference.

[17] Archambault, S., et al., ApJL, 817, L7, 2016.

[18] Lyne, A. G., et al., MNRAS, 451, 581, 2015.

[19] Archer, A., et al., ApJ, 821, 129, 2016.

[20] Benbow, W. for the VERITAS Collaboration. This conference.

[21] Shahinyan, K. for the VERITAS Collaboration. This conference.

[22] Pueschel, E. for the VERITAS Collaboration. This conference.

[23] Abeysekara, A. U., et al., ApJL, 815, L22, 2015.

[24] Pacciani, L., The Astronomer's Telegram, 7402, 2015. 


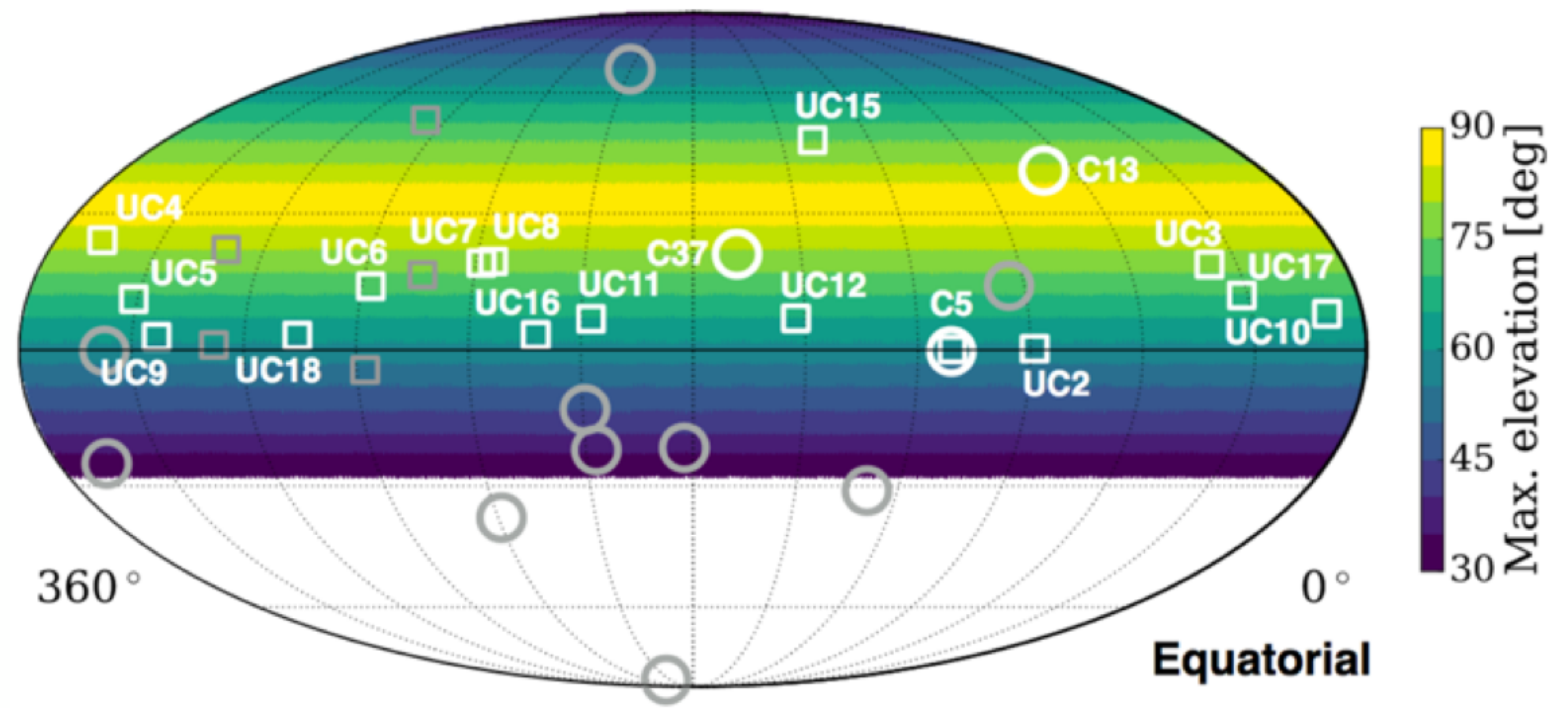

FIGURE 10. IceCube neutrino locations observed by VERITAS. The labeled events all have relatively small angular uncertainty (muon track events, with $\sim 1^{\circ}$ angular resolution) and a high probability ( $>40 \%$ ) of astrophysical origin [34].

[25] Mirzoyan, R., The Astronomer's Telegram, 7416, 2015.

[26] Holder, J., et al., ApJL, 583, L9, 2003.

[27] Krawczynski, H., et al., ApJ, 601, 151, 2004.

[28] Mukherjee, R., The Astronomer's Telegram, 8148, 2015.

[29] Zitzer, B., for the VERITAS Collaboration, in Proc. 34 ${ }^{\text {th }}$ ICRC, (The Hague, The Netherlands, 2015), arXiv:1509.01105.

[30] Geringer-Sameth, A., Koushiappas, S. M., \& Walker, M. G., Phys. Rev. D., 91, 083535, 2015.

[31] Staszak, D., for the VERITAS Collaboration, in Proc. 34 ${ }^{\text {th }}$ ICRC, (The Hague, The Netherlands, 2015), arXiv:1508.06597.

[32] Abeysekara, A. U., et al., ApJL, 818, L33, 2016.

[33] Aartsen, M. G., et al., Phys. Rev. Letters, 113, 101101, 2014.

[34] Santander, M., for the VERITAS \& IceCube Collaborations, in Proc. $34^{\text {th }}$ ICRC, (The Hague, The Netherlands, 2015), arXiv::1509.00517.

[35] The IceCube Collaboration, in Proc. $34^{\text {th }}$ ICRC, (The Hague, The Netherlands, 2015), PoS(ICRC2015) 1052.

[36] Biland, A., et al., The Astronomer's Telegram, 9137, 2016.

[37] Sandoval, A. for the HAWC Collaboration. This conference.

[38] Riviere, C. for the HAWC Collaboration. This conference.

[39] Krause, M. for the VERITAS Collaboration. This conference.

[40] Acciari, V. A., et al., ApJL, 719, L69, 2010.

[41] Karpova, A., Zyuzin, D., Danilenko, A., \& Shibanov, Y., MNRAS, 453, 2241, 2015.

[42] Benbow, W. for the CTA Collaboration. This conference. 


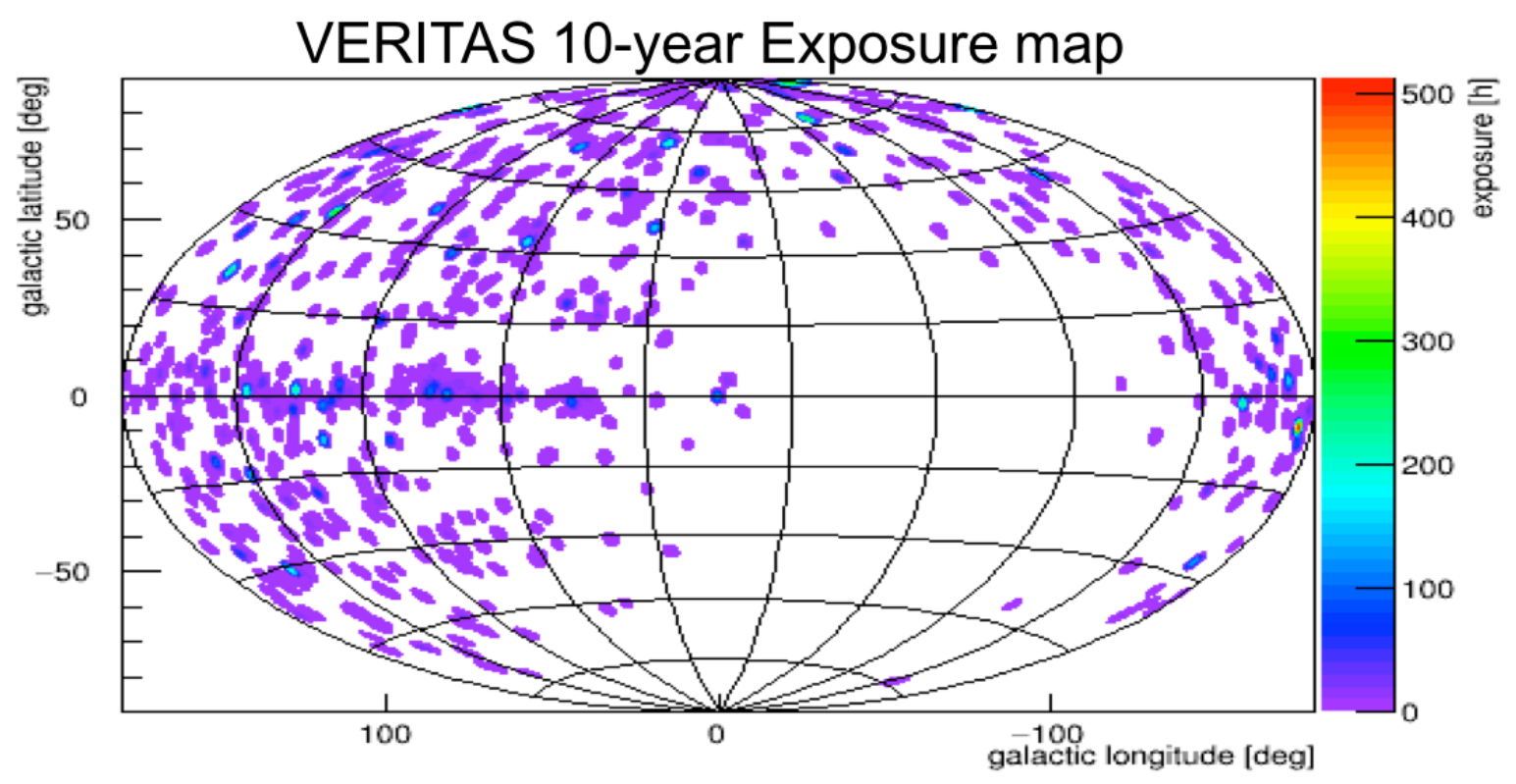

FIGURE 11. The VERITAS exposure map, after 10 years of observations.
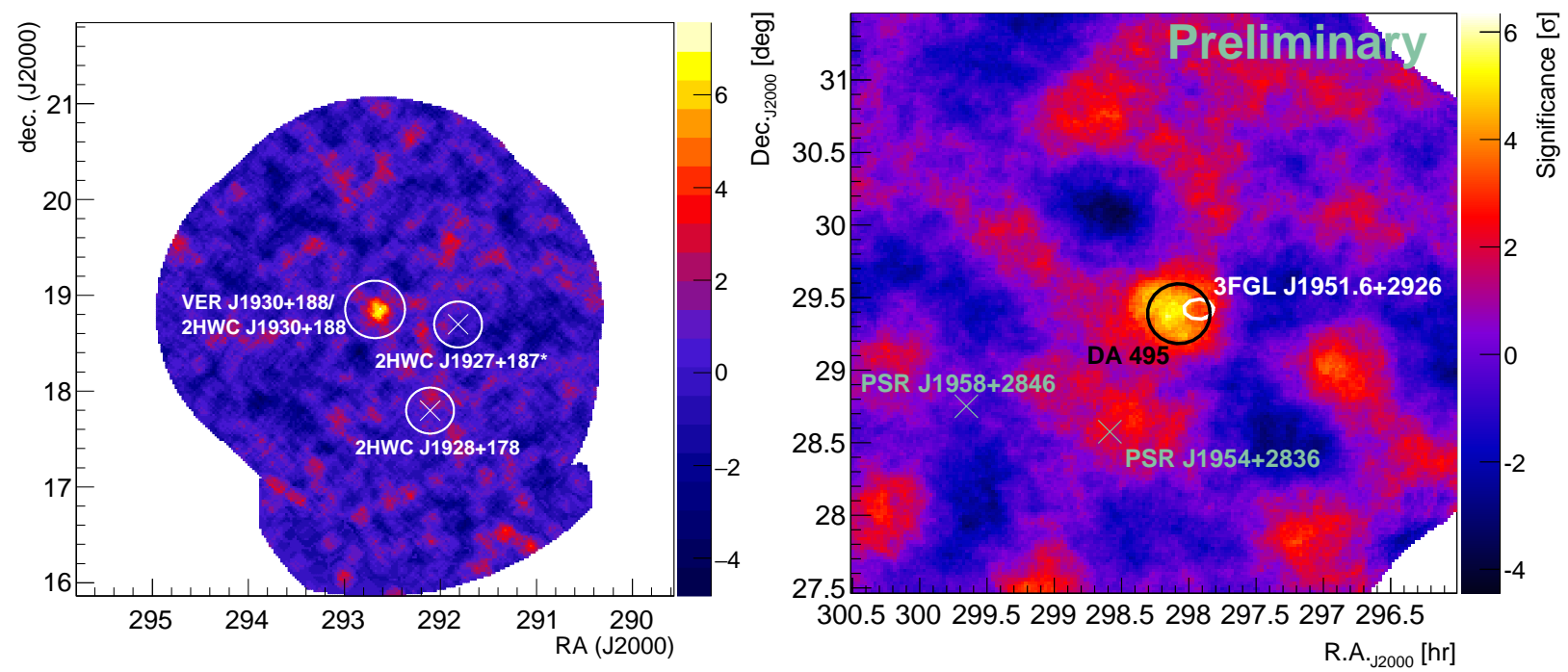

FIGURE 12. VERITAS significance sky maps for two regions containing HAWC sources from the preliminary 1-year catalog. On the left is the region around the VERITAS source VER J1930+188, likely associated with the PWN in SNR G54.1+0.3, in which three HAWC sources have been identified. White crosses indicate the two HAWC sources without a VERITAS counterpart. White circles indicate regions excluded from the background estimation. On the right is the region around another HAWC source, 2HWC J1953+294. The brightest VERITAS emission overlaps with the PWN DA 495. 